\title{
Does Psychosocial Stress Impact Cognitive Reappraisal? Behavioral and Neural Evidence
}

\author{
Maheen Shermohammed ${ }^{1}$, Pranjal H. Mehta ${ }^{2}$, Joan Zhang ${ }^{1}$, Cassandra M. Brandes ${ }^{3}$, \\ Luke J. Chang ${ }^{4}$, and Leah H. Somerville ${ }^{1}$
}

\begin{abstract}
Cognitive reappraisal (CR) is regarded as an effective emotion regulation strategy. Acute stress, however, is believed to impair the functioning of prefrontal-based neural systems, which could result in lessened effectiveness of CR under stress. This study tested the behavioral and neurobiological impact of acute stress on CR. While undergoing fMRI, adult participants $(n=54)$ passively viewed or used CR to regulate their response to negative and neutral pictures and provided ratings of their negative affect in response to each picture. Half of the participants experienced an fMRI-adapted acute psychosocial stress manipulation similar to the Trier Social Stress Test, and a control group received parallel manipulations without the stressful components. Relative to the control group, the stress group exhibited heightened stress as in-
\end{abstract}

dexed by self-report, heart rate, and salivary cortisol throughout the scan. Contrary to our hypothesis, we found that reappraisal success was equivalent in the control and stress groups, as was electrodermal response to the pictures. Heart rate deceleration, a physiological response typically evoked by aversive pictures, was blunted in response to negative pictures and heightened in response to neutral pictures in the stress group. In the brain, we found weak evidence of stress-induced increases of reappraisalrelated activity in parts of the PFC and left amygdala, but these relationships were statistically fragile. Together, these findings suggest that both the self-reported and neural effects of CR may be robust to at least moderate levels of stress, informing theoretical models of stress effects on cognition and emotion.

\section{INTRODUCTION}

The ability to flexibly direct cognitive resources to influence an emotional response, known broadly as emotion regulation, is a key feature of healthy psychological functioning. A particularly well-studied strategy to regulate emotions is cognitive reappraisal (CR), which involves reinterpreting the content of an emotional stimulus in a way that changes its meaning. For example, a student with a messy roommate may try to downregulate a negative affective response to a pile of dirty dishes by remembering that her roommate is a busy medical student with no intentions of being inconsiderate.

$\mathrm{CR}$ is considered a highly effective method of reducing negative affective responses when compared with passively experiencing affect or attempting to suppress it. It is more effective at decreasing negative and increasing positive emotions (Gross, 1998) and modulating biological components of the emotional response, such as sympathetic nervous system activity and amygdala activation (Goldin, McRae, Ramel, \& Gross, 2008; Gross, 1998). In addition, using CR in everyday life predicts fewer depressive symptoms and greater psychological well-being (Gross \& John, 2003). However, the efficacy of CR has

\footnotetext{
${ }^{1}$ Harvard University, ${ }^{2}$ University of Oregon, ${ }^{3}$ Northwestern
} University, ${ }^{4}$ Dartmouth College

been primarily tested in controlled laboratory environments free of the distraction and arousal of external stressors. As such, it is less clear whether CR remains robust under more complex contexts such as stress. Stressful circumstances are precisely the time when one would benefit from intact emotion regulation, and it would therefore be especially problematic if this popular emotion regulation strategy were flimsy under such contexts. To address this question, this study evaluated the impact of acute stress on CR and its associated mechanisms.

Here, we consider stress to mean a multilayered reaction to a perceived threat, which interrupts biological homeostasis and results in a multifaceted response at subjective psychological, endocrine, and physiological levels (McEwen, 2000). Previous studies suggesting a link between stress and emotion regulatory processes have used the glucocorticoid hormone cortisol as a proxy for stress. However, the exclusive use of cortisol in the measurement of stress limits the understanding of these effects because cortisol is not a direct proxy for stress. Not all situations perceived as stressful result in a cortisol response (Hellhammer, Wüst, \& Kudielka, 2009), and cortisol increases are not uniquely provoked by events perceived as stressful (Anisman \& Merali, 1999). As such, this study aimed to offer a more comprehensive account of stress effects by measuring stress on multiple levels. 
Initial observations suggest that negative or stressful contexts influence whether someone will choose to use $\mathrm{CR}$ as well as the psychological and physiological consequences of doing so. Previous work has shown that participants were disinclined to choose to use CR while experiencing intense negative situations in the laboratory (Sheppes, Scheibe, Suri, \& Gross, 2011). In both healthy and trauma-exposed populations, greater perceived stress or stress-related symptoms are related to decreased tendencies to use CR (Miklósi, Martos, Szabó, Kocsis-Bogár, \& Forintos, 2014; Boden, Bonn-Miller, Kashdan, Alvarez, \& Gross, 2012; Moore, Zoellner, \& Mollenholt, 2008). In addition, when explicitly instructed to use CR, participants with elevated levels of cortisol are less successful at regulating conditioned fear responses (Raio, Orederu, Palazzolo, Shurick, \& Phelps, 2013) and generate fewer nonnegative reappraisals (Tsumura, Sensaki, \& Shimada, 2015). Furthermore, using CR to downregulate negative responses to laboratory stressors can paradoxically increase cortisol responding (Denson, Creswell, Terides, \& Blundell, 2014). Together, this work suggests that CR and stress interact in complex ways and that the efficacy of CR may be vulnerable while an individual is experiencing stress.

Neurobiological models indicate that CR and stress engage overlapping neural substrates, further supporting the possibility for modulatory interactions of stress on CR efficacy. CR is believed to rely on domain-general cognitive control systems largely in the PFC to modulate (usually decrease) activation in limbic regions, particularly the amygdala (Buhle et al., 2014; Ochsner \& Gross, 2005). Meanwhile, acute stress is believed to inhibit PFC, impairing the very control systems recruited in CR, while simultaneously bolstering amygdala responsiveness (Arnsten, 2009). This is reflected in the impairment of executive functions such as working memory, attention, and cognitive flexibility under stress (Olver, Pinney, Maruff, \& Norman, 2015; Luethi, Meier, \& Sandi, 2009; Alexander, Hillier, Smith, Tivarus, \& Beversdorf, 2007) and the enhancement of phenomena such as classical fear conditioning (Luethi et al., 2009) and memory for emotional pictures (Payne et al., 2007; Buchanan \& Lovallo, 2001).
The goal of this study was to evaluate the impact of acute psychological stress on CR and its associated neural substrates. Half of the participants experienced acute stress during fMRI scanning, induced by a variant of the Trier Social Stress Task, while attempting to use CR to regulate their emotional response to aversive images. The other participants experienced a control version of the stress task, which mimicked procedural aspects of the stress induction but did not induce stress. Analyses first validated the effectiveness of the stress manipulation and then measured the degree to which stress modulated emotional reactivity and CR success. We predicted that acute stress would impair CR success and that it would do so by impacting the adaptive responding of biological systems that are engaged by both CR and stress. Brain imaging data were used to identify stress-related modulation of activity in the neural circuitry previously implicated in CR.

\section{METHODS}

\section{Participants}

Fifty-six young adults aged 18-23 years participated in this study. Two participants were excluded from analyses because of noncompliance, leaving a final sample of 54 (see Table 1 for detailed demographic information). All participants were right-handed, nonsmokers, proficient in English, and not currently receiving treatment for psychological or neurological disorders. In an effort to recruit a more representative sample, no more than $25 \%$ of the participants were current or former students at Harvard University. All participants provided informed written consent for their participation. Research procedures were approved by the Committee on the Use of Human Subjects at Harvard University and by the Army Human Research Protections Office.

\section{Tasks}

\section{Task Overview}

The goal of the experimental session was to elicit a stress response that would persist throughout fMRI scanning to

Table 1. Demographics of Assigned Groups

\begin{tabular}{llrr}
\hline & Control & Stress & Statistic \\
\hline Total & 25 & 29 & $\chi^{2}(1)=0.074, p=.785$ \\
Female & $48 \%$ & $51.72 \%$ & $\chi^{2}(1)=0.074, p=.785$ \\
White (non-Hispanic) & $48 \%$ & $51.72 \%$ & $t(50.53)=-0.510, p=.613$ \\
Age & $20.89(1.71)$ & $21.13(1.67)$ & $t(50)=-0.800, p=.427$ \\
IQ & $116.12(12.83)$ & $118.86(12.22)$ & $t$ \\
\hline
\end{tabular}

For age and IQ, means and standard deviations are reported, and all $t$ tests are Welch's $t$ tests for unequal variances. IQ was obtained using the Wechsler Abbreviated Scale of Intelligence (Second Edition) FSIQ-2 score. 
Figure 1. Study design. (A) Three indices of stress were acquired repeatedly throughout the experiment, denoted by checkmarks. The gray box indicates which measures occurred in the MRI. (B) Example screens from the math task for the stress and control groups. For the stress group, a blue bar growing quickly from the left to right sides of the screen indicated the time remaining to answer the math problem, and arrows falsely indicated whether participants (bottom arrow) and their peers (top arrow) were performing below average (red), average (yellow), or above average (green). The control task had similar visual features but with simpler math problems, no time pressure, and no social comparison.

(C) The CR task first instructed participants to use CR (i.e., Decrease) or Look at the forthcoming picture, then displayed an image, and then acquired self-reported affect ratings on a scale of 1 ("not at all bad") to 5 ("very bad").

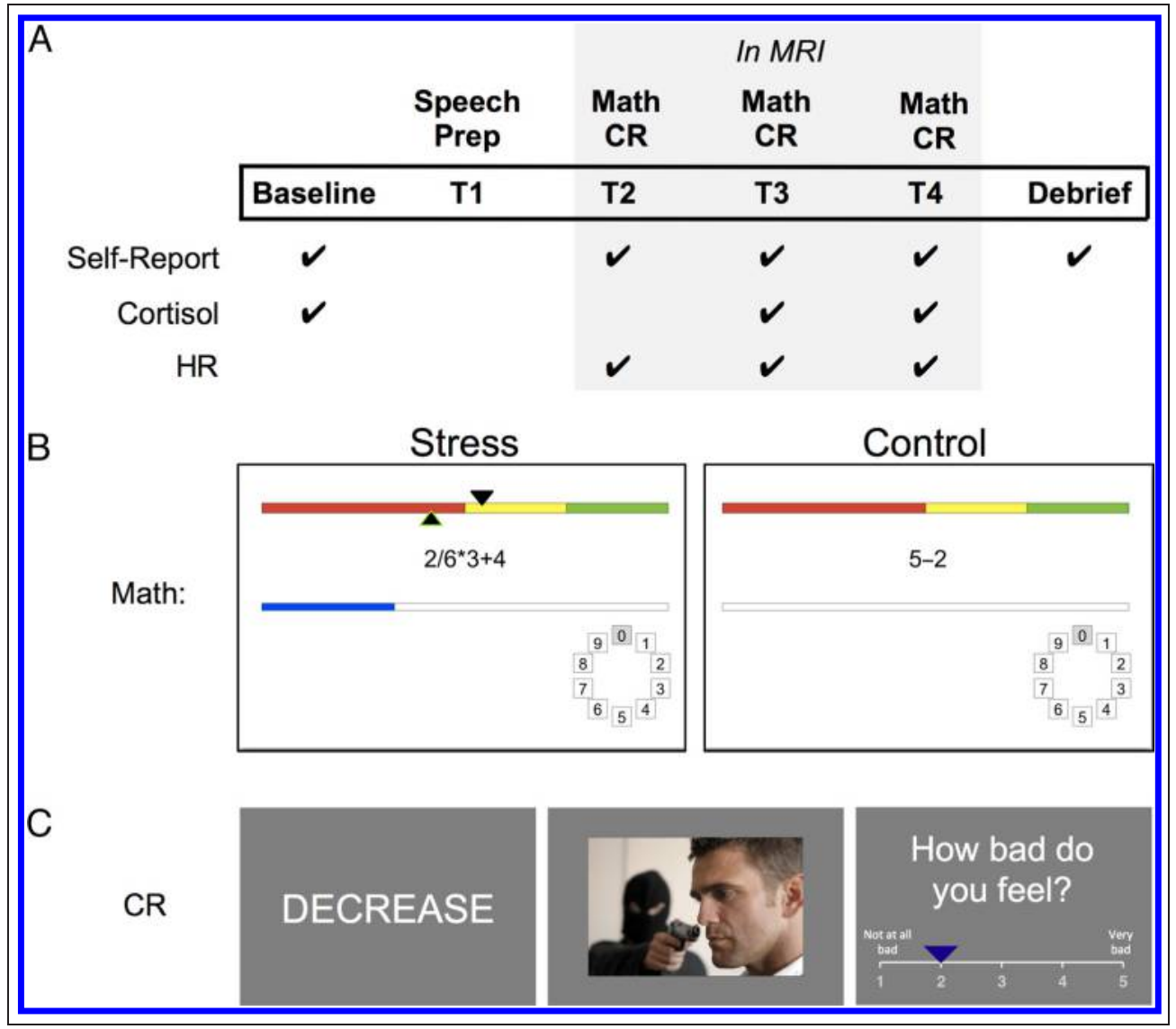

observe effects of stress on reappraisal processes. To do so, participants in the Stress condition completed elements of established stress induction tasks immediately before and during fMRI scanning. Participants in the Control condition completed highly similar procedures, absent the stressful components. During fMRI scanning, the primary dependent variables were behavioral and neural activity measured during the CR task. In addition, stress indices were acquired throughout fMRI scanning, including saliva samples for cortisol analysis, self-reported affect, and heart rate (HR; see Figure 1A). These measures were used for manipulation checks of the stress induction and for individual difference analyses.

\section{CR Task}

Building on prior work, we employed a task frequently used to target CR processes (Ochsner et al., 2004). In this task, participants viewed negative and neutral images and were asked to use CR to decrease their emotional response to half of the negative images (Figure 1C). Before the MRI session, participants were thoroughly trained on the strategy of CR. They were given example reappraisals for sample negative images (e.g., "help is on the way," "it's just a scene from a movie") and generated their own reappraisals aloud to the experimenter over several practice trials until they demonstrated clear understanding of the goals of the task.

During fMRI scanning, a 2-sec instructional cue first informed participants whether they were to passively view ("LOOK") or reappraise ("DECREASE") an image that followed. The image was displayed ( $8 \mathrm{sec}$ ), and then participants rated how negative they felt on a scale of $1-5$ using a button response box held in the right hand ( $2 \mathrm{sec})$. Jittered fixation periods $(2-8 \mathrm{sec})$ were presented between the image display and the rating scale and between the rating scale and the next trial. Seventy-two pictures (24 decrease-negative, 24 look-negative, 24 lookneutral) were presented over three scanning runs with 24 trials each. Run order was counterbalanced across participants. Images were from the International Affective Picture System (Lang, Bradley, \& Cuthbert, 1997) database, ${ }^{1}$ and all included depictions of people. Two sets of negative images were constructed with the same average normative valence and arousal and assigned to the decrease-negative or look-negative condition, counterbalanced across participants. In addition to self-report ratings, heart rate (HR) and electrodermal activity (EDA) were acquired as measures of emotional response to each of the three picture conditions. 


\section{Speech Stress Induction Task}

The speech stress induction task was adapted from the preparation phase of the Trier Social Stress Task (Kirschbaum, Pirke, \& Hellhammer, 1993). Before the MRI scan, participants in the stress group were told that, immediately after the scan, they would give a speech about what makes a good friend, including the participant's own personal strengths and weaknesses as a friend. The speech was to be delivered to a panel of judges who would code their verbal and nonverbal behavior and video-recorded for further evaluation. Participants in the control group were told that they would write a story immediately after the MRI scan that would not be used as data in the experiment. Both groups were given 3 min to prepare for their respective task. After the preparation period, participants entered the MRI, and three times over the course of the scanning session, they were reminded of the forthcoming speech (stress group) or story-writing (control group) task (e.g., "A few more scans and then you can come out and give that speech you prepared"; "The judges for your speech just arrived, so we'll be able to start that right after the MRI"; "Just a little while longer and then our judges will be ready to hear your presentation"). Upon leaving the MRI, participants were debriefed and informed that they would not have to make the speech or write the story.

\section{Math Stress Induction Task}

The math stress induction task was adapted from the Montreal Imaging Stress Task (Dedovic et al., 2005). During fMRI scanning, participants were presented with math problems, which they answered by selecting a number on a rotary dial (see Figure $1 \mathrm{~B}$ for a diagram). The dial began with " 0 " highlighted, and participants used the button box to navigate around the dial to select their chosen response.

For participants in the stress group, these problems included up to 4 one- or two-digit integers, and operations could include any combination of addition, subtraction, multiplication, and division (e.g., 2/6*12+4). They were given a very limited amount of time to answer these questions, and to increase the sense of urgency, they saw a progress bar growing on the screen and heard a tone of escalating frequency. This time limit changed adaptively to ensure poor performance. An initial time limit was calculated from performance speed during a nontimed practice round and then flexibly shortened or lengthened if the participant got the previous three problems correct or incorrect, respectively.

Participants in the stress condition were also given negative social evaluative feedback about their task performance. First, they were falsely told before the task that they must get at least $70 \%$ of the questions correct for researchers to be able to use their data and that most people get about $80 \%$ correct. In reality, the average per- formance for this group was 44\%. During the task itself, they saw two arrows pointing to a colored bar. They were told that the top arrow indicated the average performance of others in their peer group and that the bottom arrow indicated their own performance. The colors on the bar corresponded to above average (green), average (yellow), and below average (red). The time pressure forced participants to perform in the red, whereas the sham performance indicator for the group stayed generally within the green zone. Finally, after every run of the task, stressed participants were given feedback from the researcher expressing surprise and disappointment at their poor performance.

The control participants completed a similar version of the task that taxed mathematical processes but did not include the potentially stressful components. The control version of the task was self-paced (hence, no time pressure), and participants did not receive the social evaluative feedback. The mathematical operations were also easier, only involving adding or subtracting up to 3 onedigit numbers at a time (e.g., 5-2).

In each of three functional imaging runs, participants completed a 2-min block of the math task, which was preceded and succeeded by $30 \mathrm{sec}$ of resting baseline where the visual interface was displayed without a math problem. HR was continuously monitored during both the math and rest blocks, serving as a measure of physiological stress in response to this task.

\section{Study Procedure}

After consenting, participants were introduced to the tasks and given the opportunity to practice them. For the math task, all participants practiced the untimed, easier version. Next, to give participants time to reduce any initial stress associated with entering the laboratory environment, participants quietly watched the first $20 \mathrm{~min}$ of an episode from the Planet Earth TV series titled "Ocean Deep," a mildly positive, informative video about animals in the ocean (Denson, Mehta, \& Ho Tan, 2013). Participants then provided their first salivary cortisol sample along with self-report mood ratings (i.e., how much they currently felt "nervous," "excited," "stressed," "alert," and "happy" on scale of 1-5). Participants then received instructions about the speech (stress group) or story (control group) task and completed the 3-min preparation phase where they could outline their speech or story. At the end of the $3 \mathrm{~min}$, participants were led to the MRI room and prepared for scanning.

In the MRI scanner, participants completed three runs each of the math and CR tasks in alternating order, with math always being first. After the first math-CR task pair (T2), participants once again provided mood ratings, which were displayed on the screen in the MRI and answered using the button response box. After the second math-CR task set (T3), participants provided their second salivary cortisol sample while remaining in the MRI 
and completed mood ratings for the third time. At the very end of the MRI session (T4), participants provided the final saliva sample and completed mood ratings for the fourth time.

After exiting the MRI, participants were asked if they knew what was happening next to give them an opportunity to volunteer any suspicions about the deceptive nature of the speech task. During the full debriefing, we asked participants whether they found the speech/ story and math tasks stressful, which gave them another chance to volunteer any suspicions they had. Finally, participants completed follow-up questionnaires and assessments and provided mood ratings for the fifth time. They were then compensated for their time and thanked for their participation.

\section{Dependent Measures}

\section{Salivary Cortisol}

Salivary cortisol samples were collected by placing SalivaBio oral swabs under the tongue for 3-4 min. They were then immediately stored in Swab Storage Tubes (Salimetrics, Inc., State College, PA) at $-20^{\circ} \mathrm{C}$. To maximize the integrity of the samples, participants were instructed to refrain from exercising for $12 \mathrm{hr}$, consuming caffeine for $2 \mathrm{hr}$, eating or drinking anything but water for $30 \mathrm{~min}$, and brushing their teeth for $1 \mathrm{hr}$ before the appointment. To reduce the effects of diurnal variation, all MRI sessions were scheduled between 12:30 and 5:30 p.m. Once data collection was complete, samples were shipped frozen overnight to the Kirschbaum laboratory (Technische Universität, Dresden, Germany) for analysis with chemiluminescence immunoassay kits (IBL International, Hamburg, Germany). Samples were analyzed in singlets, and the interassay coefficient of variation was less than $8 \%$. One participant whose baseline cortisol level was almost 5 SDs above the mean was removed from any analyses that did not correct for baseline levels.

\section{Heart Rate}

HR was monitored with a fiber-optic oximetry sensor on the left ring finger using BIOPAC Systems MP150 and OXY-MRI (Nonin Medical, Inc., Plymouth, MN) hardware and AcqKnowledge software. Technical difficulties with the AcqKnowledge software resulted in full data loss for $n=8$. Any participant with at least one full run of $\mathrm{HR}$ data was included in these analyses, resulting in $n=46$ usable data sets.

These data were collected for two distinct purposes. First, HR acquired during each run of the math task was compared with the resting baseline preceding it to provide an indicator of autonomic stress responsiveness. Second, previous research has shown that viewing aversive pictures elicits a brief, parasympathetically mediated
HR deceleration, and the magnitude of this deceleration varies by stimulus intensity (Bradley, Codispoti, Cuthbert, \& Lang, 2001; Campbell, Wood, \& McBride, 1997). Thus, HR was acquired during the CR task as a physiological index of emotional responding to pictures. The pulse oximeter is not ideal for stimulus-locked measurements because, rather than providing an electrocardiogram directly, it calculates beats per minute by averaging over a window of heartbeats, causing a $~ 3.5$-sec delay between initial stimulus presentation and the resulting change in HR (Nonin Medical, Inc., personal communication). Thus, the entire HR trace was shifted by $3.5 \mathrm{sec}$ before computing the average HR for the $4.5 \mathrm{sec}$ after picture onset, corrected for the average HR during the 2 sec preceding.

\section{Electrodermal Activity}

EDA was recorded during fMRI scanning with $\mathrm{Ag}-\mathrm{AgCl}$ electrodes on the left index and middle fingers using BIOPAC Systems MP150 and EDA100C-MRI hardware and AcqKnowledge software at a sampling rate of $250 \mathrm{~Hz}$. All data were first processed with a 1-Hz low-pass filter to remove high-frequency scanner noise. In accordance with the procedure in Raio et al. (2013), the amplitude of the skin conductance response for each trial was calculated using the maximum change from base to peak in the $0.5-4.5 \mathrm{sec}$ after picture onset. Amplitudes below $0.02 \mu \mathrm{sec}$ were scored as 0 , and the data were square root transformed to normalize distributions for standard statistical analyses. Technical difficulties with the software and the exclusion of participants who did not produce any skin conductance responses resulted in a final sample size of $n=41$ usable EDA data sets.

\section{fMRI Analysis}

\section{Acquisition and Preprocessing}

Brain imaging was performed on a 3.0-T Siemens Prisma scanner with a 32-channel head coil (Siemens Medical Systems, Erlangen, Germany) at the Harvard University Center for Brain Science Neuroimaging. A T1-weighted high-resolution anatomical image of the brain was acquired using a multiecho multiplanar rapidly acquired gradient-echo sequence (176 sagittal slices, repetition time $=2200 \mathrm{msec}$, echo time $=1.67 \mathrm{msec}$, flip angle $=$ $7^{\circ}$, slice thickness $=1 \mathrm{~mm}$, voxel size $\left.=1 \times 1 \times 1 \mathrm{~mm}\right)$. Functional images were collected using an EPI T2*weighted sequence sensitive to the BOLD response (69 axial slices per whole-brain volume, voxel size $=$ $2.2 \times 2.2 \times 2.2 \mathrm{~mm}$, repetition time $=2000 \mathrm{msec}$, echo time $=35 \mathrm{msec}$, flip angle $=80^{\circ}$, multiband acceleration factor $=3$ ). Functional slices were oriented to a slightly greater tilt than the AC-PC plane to minimize signal dropout due to sinus cavities. Nonetheless, an abnormally large sinus cavity in one participant resulted in excessive 
frontal distortion. This participant was excluded from all imaging analyses.

Functional imaging data were preprocessed using the Functional MRI of the Brain Software Library (FSL, Version 5.0.4; Smith et al., 2004) tools implemented in Nipype (v. 0.11.0; Gorgolewski et al., 2011) using the Lyman interface (v. 0.0.7; www.cns.nyu.edu/ mwaskom/software/ lyman/). Each functional scan was first realigned to its middle volume, spatially smoothed with a 6-mm FWHM Gaussian kernel, and high-pass filtered at $128 \mathrm{sec}$. Functional scans were coregistered to individual-participant anatomical images using bbregister (v. 5.3.1; Greve \& Fischl, 2009), and all first level or individual-participant analyses were conducted in this native space. Analyses comparing participants or groups first normalized statistical maps to a Montreal Neurological Institute (MNI) brain template using linear and nonlinear warping methods through the Advanced Normalization Tools software (v. 1.9.x; Avants, Tustison, \& Song, 2009).

\section{Modeling of CR Task}

Preprocessed images were entered into a standard general linear model in FSL, which estimated neural responses to the cue period (collapsed over trial types), the three types of picture periods (decrease-negative, look-negative, and look-neutral), and the rating period (collapsed over trial types). Regressors used boxcar functions convolved with the canonical double-gamma hemodynamic response function implemented in FSL. The model also included nuisance regressors for motion parameters, temporal derivatives for each regressor of interest, and temporal filter regressors with a cutoff of $128 \mathrm{sec}$. To remove additional noise, functional volumes with motion greater than $1 \mathrm{~mm}$ or whole-brain intensity values greater than 4.5 SDs away from the mean were censored from the model as additional regressors; no scan had greater than 10\% censored volumes. All participants had at least two of three scans of usable data.

\section{ROI Analysis}

Unbiased, a priori ROIs were identified using a previous meta-analysis of 48 neuroimaging studies of reappraisal (Buhle et al., 2014). Eleven 6-mm spheres were centered on activation peaks from the reappraise $>$ emotional baseline contrast reported in the meta-analysis, in addition to two 4-mm spheres in the bilateral amygdalae from the emotional baseline $>$ reappraise contrast reported in the meta-analysis (see Table 2 for ROI coordinates). Mean parameter estimates were extracted for the picture period from each ROI and tested for task and stress effects. $p$ Values from these tests were corrected for

Table 2. ROI Analyses for the Effects of Stress on Reappraisal and Reactivity

\begin{tabular}{|c|c|c|c|c|c|c|c|c|}
\hline \multirow{3}{*}{$\frac{R O I}{\text { Inferior frontal gyrus }}$} & \multirow{3}{*}{$\frac{\text { Side }}{\mathrm{R}}$} & \multicolumn{3}{|c|}{ MNI Coordinates } & \multirow{2}{*}{\multicolumn{2}{|c|}{ 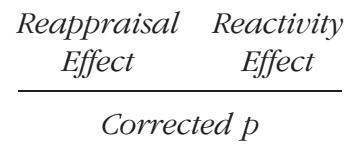 }} & \multirow{2}{*}{\multicolumn{2}{|c|}{$\frac{\text { Reappraisal } \times \text { Group } \text { Reactivity } \times \text { Group }}{\text { Corrected } p(\text { Uncorrected } p)}$}} \\
\hline & & \multirow{2}{*}{$\frac{x}{60}$} & \multirow{2}{*}{$\frac{y}{24}$} & \multirow{2}{*}{$\frac{z}{3}$} & & & & \\
\hline & & & & & $<.001 * *$ & .624 & $.643(.346)$ & $.909(.198)$ \\
\hline Middle frontal gyrus & $\mathrm{R}$ & 51 & 15 & 48 & $<.001 * *$ & $.010 *$ & $.291(.045 *)$ & $.909(.282)$ \\
\hline Medial frontal gyrus & $\mathrm{R}$ & 9 & 30 & 39 & $<.001 * *$ & .219 & $.643(.334)$ & $.909(.837)$ \\
\hline Anterior cingulate gyrus & $\mathrm{L}$ & -3 & 24 & 30 & $<.001 * *$ & .824 & $.936(.870)$ & $.909(.531)$ \\
\hline Superior frontal gyrus & $\mathrm{L}$ & -9 & 12 & 69 & $<.001 * *$ & .824 & $.936(.872)$ & $.909(.454)$ \\
\hline Middle frontal gyrus & $\mathrm{L}$ & -33 & 3 & 54 & $<.001 * *$ & $<.001 * *$ & $.643(.161)$ & $.909(.909)$ \\
\hline Anterior insula & $\mathrm{L}$ & -36 & 21 & -3 & $<.001 * *$ & $.032 *$ & $.936(.936)$ & $.909(.815)$ \\
\hline Inferior frontal gyrus & $\mathrm{L}$ & -42 & 45 & -6 & $<.001 * *$ & .219 & $.643(.288)$ & $.909(.530)$ \\
\hline Superior temporal gyrus & $\mathrm{R}$ & 63 & -51 & 39 & $<.001 * *$ & .942 & $.804(.586)$ & $.531(.041 *)$ \\
\hline Angular gyrus & $\mathrm{L}$ & -42 & -66 & 42 & $<.001 * *$ & .792 & $.804(.618)$ & $.909(.776)$ \\
\hline Middle temporal gyrus & $\mathrm{L}$ & -51 & -39 & 3 & $<.001 * *$ & .135 & $.729(.449)$ & $.909(.612)$ \\
\hline Amygdala & $\mathrm{R}$ & 30 & -3 & -15 & .291 & .219 & $.643(.249)$ & $.909(.341)$ \\
\hline Amygdala & $\mathrm{L}$ & -18 & -3 & -15 & .122 & .824 & $.202(.016 *)$ & $.909(.884)$ \\
\hline
\end{tabular}

Each ROI was subject to a separate ANOVA testing for the effect of stress group on reappraisal or reactivity. Significance tests for each interaction are reported both with and without correction for the number of ROIs tested. Region labels were based on the Harvard-Oxford cortical and subcortical atlases cross-referencing (Mai, Paxinos, \& Voss, 2008).

$* p<.05$.

$* * p<.001$. 
13 comparisons by controlling for the false discovery rate using the Benjamini-Hochberg method.

\section{Whole-brain fMRI Analysis}

To supplement ROI analyses, a random effects analysis focused on the picture period was also performed across the whole brain. First, to corroborate established findings with this task, one-tailed $t$ tests for contrasts isolating the effects of reappraisal (decrease-negative $>$ look-negative) and emotional reactivity (look-negative $>$ look-neutral) were computed across the whole sample. Next, twosample $t$ tests comparing the stress and control groups were computed for these contrasts to identify activations that were significantly greater in the stress versus control groups.

To address individual differences in neural responses in reaction to stress reactivity measures, we conducted three separate, group level models using HR changes, cortisol changes, or self-reported stress levels as continuous covariates of interest. All contrast maps were corrected at a FWE threshold of $p<.05$ using FSL's cluster-based correction. Activation maps were first thresholded $(z>2.3)$, and then Gaussian Random Field theory was used to calculate a cluster size threshold, below which clusters were removed.

\section{Analysis Strategy}

Primary analyses evaluated how stress influenced responses during the reappraisal task. On one hand, stress could affect a participant's ability to use reappraisal effectively. If this was the case, stress should specifically reduce any differences between the decrease-negative and look-negative conditions, that is, the "reappraisal contrast." Another possibility is that stress could make participants more reactive to negative pictures in general. This would result in an exacerbation of any differences between the look-negative and look-neutral conditions, that is, the "reactivity contrast."

Although the stress group experienced more stress than the control group on average, some participants in the control group reported feeling stressed, and some in the stress group did not. We thus decided to proceed with two parallel analysis streams. In the first, we considered the stress measure categorically based on the experimental manipulation of stress, comparing those who were stressed by the stress manipulation relative to unstressed controls. Therefore, we excluded any "nonresponders" in the stress group (i.e., those who at no point during the manipulation rated their stress level as higher than they did at baseline, $n=5$ ) and any stressed participants in the control group (i.e., those whose average stress rating increase from baseline was greater than 0.67 , which was 1.5 times the interquartile range above the upper quartile, $n=2$ ).
In the second analysis stream, we considered stress on a continuum, including all participants irrespective of their response levels or which experimental manipulation they received. This analysis examined the effects of how much stress someone was feeling during fMRI scanning relative to baseline. We computed three separate measures of stress levels using self-report, HR, and cortisol. To index an individual's self-reported stress levels, we computed the average of the "stressed" mood rating for T2, T3, and T4, corrected for the rating at baseline (T1). As another measure of stress, we calculated the difference between the average HR for the period of the math task when participants were answering problems and the rest period preceding it (HRdiff). Finally, we assessed cortisol levels using the formula in Pruessner, Kirschbaum, Meinlschmid, and Hellhammer (2003) for area under the curve with respect to the increase, which is a measure of a participant's cortisol levels throughout the experiment correcting for the pretask baseline level.

For both analysis streams, we conducted two-way ANOVAs to test the effects of stress and the reappraisal contrast (decrease-negative vs. look-negative) or reactivity contrast (look-negative vs. look-neutral) on the outcome variables: picture ratings, neural activation, and physiology during the CR task. All statistical analyses were performed in R 3.2.3 (R Core Team, 2015).

\section{RESULTS}

\section{Stress Induction}

Overall, the speech and math tasks were successful in inducing stress, as assessed by self-report, cortisol levels, and HR changes. Cortisol levels and self-reported stress were both expected to increase from baseline levels measured at the beginning of the study. Both of these measures were submitted to a two-factor ANOVA, using Group (Stress, Control) as a between-participant factor and Sample number (ordered time points) as a withinparticipant factor. The distribution of cortisol values was right-skewed and thus $\log _{10}$ transformed first. There was a significant Group $\times$ Sample number interaction for self-reported stress ratings $(F(4,50)=12.89, p<.001)$, which subsequent $t$ tests revealed was driven by no difference between groups during the initial baseline measure $(p=.90)$ or after participants were debriefed $(p=$ .58 ), and an increase in ratings for the stress group for samples T2-T4 compared with the control group ( $p$ s $<$ .001 ; Figure 2A).

There was also a significant interaction between group and sample for cortisol responding $(F(2,101)=3.880$, $p=.024)$, with no difference at baseline $(p=.43)$ and an increase for the stress group at samples T3-T4 compared with controls $(p s<.005)$. HR levels were expected to increase during the math task relative to the fixation period preceding it. A two-factor ANOVA comparing the average HR during the math and baseline fixation periods 


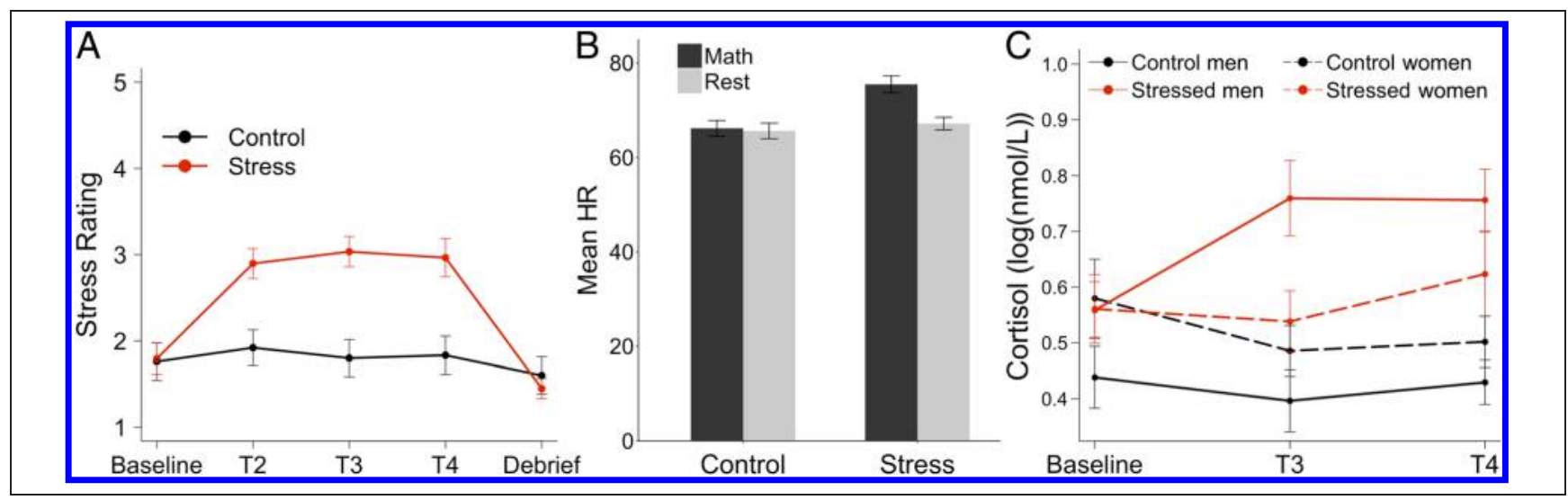

Figure 2. Measures of components of the stress response in the stress and control groups. (A) Participants in the stress group exhibited higher average self-reported stress ratings after the stress induction (T2-T4), but not at baseline or after debriefing. (B) Participants in the stress group showed an increase in average HR during the math task compared with the rest period preceding it, whereas the control participants did not. (C) Participants in the stress group exhibited increased cortisol from baseline after the stress induction, although this effect was greater in male participants. Cortisol values are log transformed. Error bars represent SEM.

between the two groups revealed a significant interaction between Group and Math task period $(F(1,46)=37.52$, $p<.001)$ driven by an increase in HR when answering problems in the stress but not control group (Figure 2B). Together, these analyses provide converging evidence that the task procedures were effective at inducing a multifaceted stress response that was selective to the stress group.

Previous studies have shown that men exhibit a greater cortisol response to laboratory stressors than women (Childs, Dlugos, \& De Wit, 2010; Kirschbaum, Kudielka, Gaab, Schommer, \& Hellhammer, 1999; Kirschbaum, Wust, \& Hellhammer, 1992). We thus also examined the interaction between group and sample separately in men and women (Figure 2C). In line with previous work, the interaction was significant in men $(F(2,50)=4.842$, $p=.012)$ but not women $(F(2,47)=0.898, p=.414)$, despite both showing increases in subjective stress (men: $F(4,99)=8.493, p<.001$, women: $F(4,99)=5.738, p<$ $.001)$.

During debriefing, 4 of the 29 participants assigned to the stress manipulation expressed suspicion about at least some component of the study (e.g., they did not believe they would have to give a speech at the end). Removing these participants' data did not impact the results of the key behavioral analyses discussed below.

\section{Effects of Stress on Emotional Reactivity and CR}

To evaluate the general effectiveness of the stimuli and task instructions, we first evaluated the main effects of Picture valence and Reappraisal instruction on affect ratings. As expected, the negative pictures were rated significantly more negatively than the neutral pictures $(F(1$, $45)=681.66, p<.001$; look-negative: mean $=2.95$, $S E M=0.075$; look-neutral: mean $=1.27, S E M=0.03)$. A significant main effect of Picture condition $(F(1,45)=$ $75.36, p<.001)$ indicated that, consistent with prior work, participants successfully reduced their negative reaction to negative pictures using reappraisal strategies (decrease-negative: mean $=2.27, S E M=0.075)$ compared with passive viewing.

The critical tests evaluated whether stress modulated reappraisal success and reactivity levels. Evaluating interactions with the stress group revealed no significant interaction between Group and Picture type on either reappraisal success $(F(1,45)=1.306, p=.259)$ or emotional reactivity $(F(1,45)=1.266, p=.266)$. Thus, assignment to the stress or control group did not influence these target emotional measures (Figure 3).

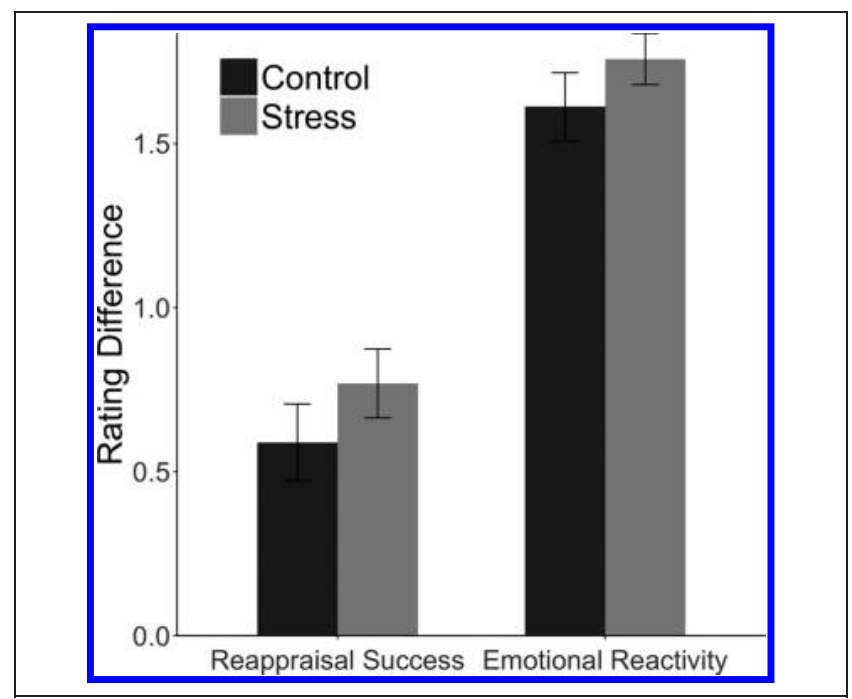

Figure 3. Reappraisal success and emotional reactivity in the stress and control groups. Reappraisal success is defined as the difference in selfreported affect ratings for look-negative $>$ decrease-negative pictures. Emotional reactivity is defined as the difference in self-reported affect ratings for look-negative $>$ look-neutral pictures. There was no effect of stress group on either measure. Error bars represent SEM. 
Secondary analyses treating stress as a continuous variable assessed whether individual differences in engagement of the components of the stress response (self-report, cortisol, and HR) influenced emotional reactivity or reappraisal. We found a significant interaction between Emotional reactivity and Self-reported stress $(F(1$, $52)=4.435, p=.04)$ and a trending interaction between reactivity and HRdiff $(F(1,44)=2.921, p=.094)$. Greater self-reported stress and a larger math-induced HR increase were associated with more negative affect ratings to look-negative pictures compared with look-neutral pictures. However, there was no effect of stress on decreasenegative pictures. There was no interaction between HRdiff and Reappraisal success $(F(1,44)=1.731, p=$ .195) and a trending interaction between Self-reported stress and Reappraisal success $(F(1,52)=3.898, p=$ $.054)$. The trending interaction was driven by an increase in response to look-negative pictures with greater stress $(r(52)=0.357, p=.008)$, whereas the response to decrease-negative was unaffected by stress $(r(52)=$ $.084, p=.547)$. We found no interaction between cortisol increases and emotional reactivity $(F(1,51)=0.345, p=$ $.559)$ or reappraisal success $(F(1,51)=0.656, p=.422)$.

\section{Effects of Stress on Neural Signatures of Emotional Reactivity and $\mathbf{C R}$}

\section{ROI Analysis}

Parallel to the behavioral analyses above, separate ANOVAs were conducted on each ROI testing for the main effects of Task condition and interactions between Task condition and Group on fMRI responses. Results are summarized in Table 2 . Consistent with meta-analytic evidence localizing the neural correlates of reappraisal, all cortical ROIs exhibited greater activation during reappraisal than while passively viewing negative images. Contrary to our predictions, no ROI showed a significant interaction between group (stress, control) and reappraisal condition after correcting for multiple comparisons. To provide the most comprehensive description of the data, we also report uncorrected results. Without correction, there was a significant interaction between
Group and Reappraisal condition in the left amygdala and the right middle frontal gyrus, with the stress group exhibiting greater reappraisal-related activity in these regions.

Contrary to our expectations, we did not find any main effects of Emotional reactivity in the amygdala ROIs. However, as discussed below, partially overlapping portions of the amygdalae were activated when examining the reactivity contrast in the whole brain. Like the reappraisal contrast, no interactions between group and reactivity condition survived correction for multiple comparisons.

We also examined whether individual differences in components of the stress response (irrespective of group) predicted reappraisal success or reactivity levels. We found no significant interactions between any of the continuous participant level stress measures and the reappraisal or reactivity contrasts. Without correction, there was a significant increase in reappraisal-related activity in the right middle frontal gyrus as HRdiff increased $(F(1$, $43)=6.782, p=.013$ ) and a trending increase in emotional reactivity in the right amygdala as HRdiff increased $(F(1,43)=3.666, p=.062)$.

\section{Whole-brain Analysis}

We supplemented targeted ROI analyses with exploratory whole-brain comparisons. Consistent with prior work (Buhle et al., 2014), we found that reappraisal (decreasenegative $>$ look-negative) recruited an extensive network of lateral and medial prefrontal regions as well as areas in the posterior temporal and parietal cortex (Figure 4, Table 3). Emotional reactivity (look-negative $>$ lookneutral) recruited regions consistent with prior work, including the bilateral amygdala and insula (Table 3).

Examining the effects of group (stress, control) on the reactivity contrast revealed a group difference in the left superior temporal gyrus, such that a prominent deactivation in the control group when viewing negative pictures was not evident in the stress group. There were no differences between groups for the reappraisal contrast. Whole-brain analyses examining different components
Figure 4. Whole-brain fMRI analyses examining reappraisal (decrease-negative $>$ looknegative) revealed the extensive network of prefrontal, temporal, and posterior parietal regions expected from previous work (Buhle et al., 2014). Images are $p<.05$, FWE corrected, and have been further thresholded at $z>5$ for visualization purposes.

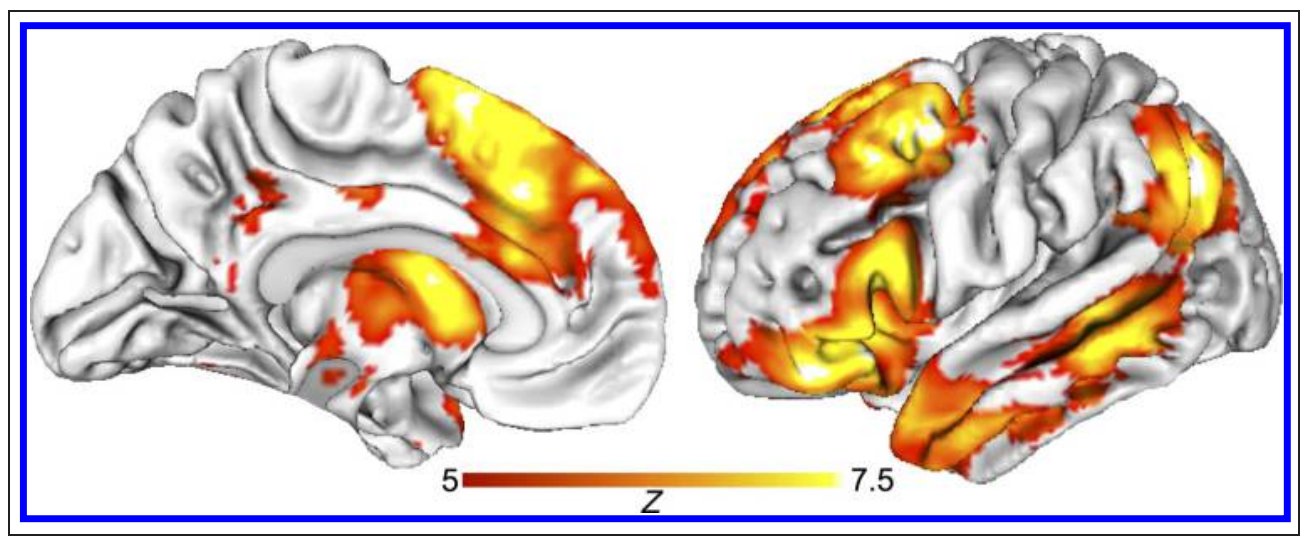


Table 3. Brain Regions Recruited during CR Task

\begin{tabular}{|c|c|c|c|c|c|c|}
\hline \multirow[b]{2}{*}{ Region } & \multirow[b]{2}{*}{ Side } & \multirow[b]{2}{*}{ Extent } & \multirow[b]{2}{*}{$\operatorname{Max} Z$} & \multicolumn{3}{|c|}{ MNI Coordinates } \\
\hline & & & & $x$ & $y$ & $z$ \\
\hline \multicolumn{7}{|c|}{ Reappraisal contrast (decrease-negative $>$ look-negative) } \\
\hline Middle frontal gyrus & $\mathrm{L}$ & 113,868 & 8.16 & -44 & 4 & 50 \\
\hline Lateral occipital cortex & $\mathrm{L}$ & & & -52 & -62 & 34 \\
\hline Superior frontal gyrus & $\mathrm{L}$ & & & -4 & 10 & 62 \\
\hline Caudate & $\mathrm{R}$ & & & 14 & 8 & 16 \\
\hline Inferior frontal gyrus & $\mathrm{L}$ & & & -46 & 24 & -6 \\
\hline Temporal pole & $\mathrm{R}$ & & & 44 & 10 & -34 \\
\hline Caudate & $\mathrm{L}$ & & & -16 & 4 & 18 \\
\hline Middle temporal gyrus & $\mathrm{L}$ & & & -58 & -40 & -6 \\
\hline Insula & $\mathrm{R}$ & & & 36 & 22 & -6 \\
\hline Inferior frontal gyrus & $\mathrm{R}$ & & & 48 & 30 & -8 \\
\hline Middle temporal gyrus & $\mathrm{R}$ & & & 50 & -38 & 2 \\
\hline Superior temporal gyrus & $\mathrm{L}$ & & & -40 & -52 & 24 \\
\hline Insula & $\mathrm{L}$ & & & -32 & 24 & -2 \\
\hline Temporal pole & $\mathrm{L}$ & & & -50 & 14 & -24 \\
\hline Superior frontal gyrus & $\mathrm{R}$ & & & 10 & 20 & 58 \\
\hline Superior temporal gyrus & $\mathrm{R}$ & & & 50 & -54 & 32 \\
\hline \multicolumn{7}{|c|}{ Reactivity contrast (look-negative $>$ look-neutral) } \\
\hline Inferior temporal gyrus & $\mathrm{L}$ & 56,664 & 8.29 & -42 & -76 & -8 \\
\hline Inferior temporal gyrus & $\mathrm{R}$ & & & 50 & -72 & 2 \\
\hline Fusiform gyrus & $\mathrm{L}$ & & & -38 & -44 & -18 \\
\hline Fusiform gyrus & $\mathrm{R}$ & & & 42 & -44 & -20 \\
\hline Precentral gyrus & $\mathrm{L}$ & & & -44 & 2 & 28 \\
\hline Middle frontal gyrus & $\mathrm{L}$ & & & -28 & -6 & 48 \\
\hline Medial frontal gyrus & $\mathrm{L}$ & & & -8 & 14 & 48 \\
\hline Habenula & $\mathrm{L}$ & & & -4 & -28 & -2 \\
\hline Thalamus & $\mathrm{R}$ & & & 18 & -30 & 4 \\
\hline Precentral gyrus & $\mathrm{R}$ & & & 42 & 6 & 26 \\
\hline Thalamus & $\mathrm{L}$ & & & -14 & -30 & 4 \\
\hline Habenula & $\mathrm{R}$ & & & 6 & -28 & -4 \\
\hline Insula & $\mathrm{L}$ & & & -28 & 20 & 2 \\
\hline Inferior frontal gyrus & $\mathrm{L}$ & & & -40 & 30 & 18 \\
\hline Superior parietal lobule & $\mathrm{L}$ & & & -28 & -48 & 44 \\
\hline Superior parietal lobule & $\mathrm{R}$ & & & 30 & -52 & 50 \\
\hline Insula & $\mathrm{R}$ & & & 30 & 26 & -2 \\
\hline Amygdala & $\mathrm{L}$ & & & -26 & -4 & -20 \\
\hline Amygdala & $\mathrm{R}$ & & & 28 & -2 & -18 \\
\hline
\end{tabular}

For both the reappraisal and reactivity contrasts, resulting maps contained one large cluster spanning many regions. Local maxima in these regions are reported as subpeaks. Region labels were based on the Harvard-Oxford cortical and subcortical atlases cross-referencing (Mai et al., 2008). 
of the stress response as continuous covariates revealed that, as HRdiff increased, activation increased for the reactivity contrast in the lingual gyrus (peak at MNI coordinates: $x=-12, y=-68, z=-2,418$ voxels) and decreased for the reappraisal contrast in the posterior cingulate cortex ( $x=4, y=-46, z=10$, 952 voxels). No brain regions survived corrections for whole-brain analyses using self-reported stress or cortisol increase as continuous covariates.

\section{Effects of Stress on Physiological Signals of Emotional Reactivity and CR}

\section{EDA Response to Pictures}

There were no significant main effects of Reappraisal or Reactivity on EDA and no interactions with Group or any of the continuous stress measures $(p s>.1)$.

\section{HR Response to Pictures}

As expected, there was a prominent decrease in HR when viewing negative pictures. This effect was attenuated when participants used reappraisal, as suggested by a significant main effect of picture type for the reappraisal contrast $(F(1,39)=6.003, p=.019)$. There was also a significant main effect of Group for the reappraisal contrast, such that participants in the stress group showed a blunted HR deceleration to negative images, whether they were reappraised or not $(F(1,39)=4.439, p=$ .042). A significant interaction between Group and Picture type for the reactivity contrast suggested that, although HR deceleration was specific to negative images in the control group, participants in the stress group continued to exhibit similar HR decelerations when viewing neutral pictures $(F(1,39)=5.891, p=.02)$.

Examining stress as a continuous measure largely mirrored the reactivity difference between groups, with interactions between both self-reported stress $(F(1,44)=$ $4.85, p=.033)$ and HRdiff $(F(1,44)=15.956, p<.001)$ and picture type in the reactivity contrast suggesting that, the more stressed a participant was, the more his or her HR decelerated in response to neutral compared with negative pictures. In addition, both of these continuous stress measures exhibited a significant main effect on HR deceleration within the reappraisal contrast (self-report: $F(1,44)=4.86, p=.033$; HRdiff: $F(1,44)=6.435$, $p=.015)$, such that, as participants were more stressed, they experienced less of an HR deceleration in response to negative pictures that were only looked at as well as those that were reappraised. Cortisol levels did not exhibit either of the aforementioned effects.

\section{DISCUSSION}

This study sought to examine the effects of stress on CR and its associated neural mechanisms. The manipulation successfully induced a rich, multifaceted stress response: The stress group exhibited an increase in cortisol, HR, and self-reported stress levels relative to the control group. Participants also responded to the emotion regulation task as expected. CR attenuated participants' emotional responses to negative images and recruited the extensive network of prefrontal, temporal, and posterior parietal brain regions that has been implicated in previous work.

Nonetheless, the observed neural and behavioral effects of stress on CR were minimal. Although we found some evidence that, the more stressed participants were, the more negative affect they experienced in response to negative images, the degree to which participants successfully decreased negative affect using CR did not vary with stress. Stress similarly did not influence electrodermal response to reappraised images. However, it is difficult to interpret the electrodermal effects because we also did not observe the expected increase in response to negative images seen in prior work (Cuthbert, Bradley, \& Lang, 1996; Lang, Greenwald, Bradley, \& Hamm, 1993). These findings should therefore be interpreted with caution. In examining neural response patterns, we found that, in general, stress exerted minimal modulatory influence on neural activity patterns both in a priori ROIs previously implicated in reappraisal processes and across the whole brain. We observed modest stress modulation of reappraisal-related activity in the left amygdala and the right middle frontal gyrus, but these observations did not survive correction for multiple comparisons.

We also measured participants' HR deceleration response while viewing the emotional and neutral images as an index of emotional responding and tested whether it varied depending on stress. This measure is an index of a parasympathetic orienting response akin to fear bradycardia, and its magnitude increases with stimulus intensity (Bradley et al., 2001; Campbell et al., 1997). Stress was associated with greater deceleration to neutral pictures and blunted deceleration to negative pictures, including negative pictures that were reappraised. Previous work has suggested that stress may induce a state of hypervigilance characterized by a heightened sensitivity to emotional stimuli at the cost of specificity (Cousijn et al., 2010; van Marle, Hermans, Qin, \& Fernández, 2009). The present findings are consistent with this idea that stress reduces the differentiation between threat- and non-threat-related stimuli in physiological measures such as HR. It should be noted, however, that other measures of emotional responding (EDA and self-reported affect) did not yield this pattern.

These results ran counter to our hypotheses. CR and stress are believed to engage overlapping neural systems, and previous behavioral work has suggested that these processes interact to influence behavior and physiology (Tsumura et al., 2015; Denson et al., 2014; Raio et al., 2013). We therefore predicted that stress would reduce the effectiveness of CR by negatively impacting the functioning 
of biological systems that are jointly modulated by CR and stress. In the following paragraphs, we explore possible reasons why CR was largely robust to the effects of stress in this study and offer directions for future work that would build a more nuanced account of the relationship between stress and emotion regulation.

In this study, we focused on psychological stressors. Although stress in its original and most fundamental conception is a nonspecific response (Selye, 1987), it is possible that components of the response that depend on the specific nature of the stressor play a more central part in influencing emotion regulation. For instance, previous work using a physical rather than psychological stressor has found evidence of stress modulation on regulatory processing (Raio et al., 2013). The intensity of induced stress may also play an important role: As with most laboratory-based studies, the intensity of stress achieved in this study is moderate. Thus, it remains unclear whether these findings would generalize to stressors of high intensity.

In addition, we were interested in eliciting and examining an ecologically valid, multifaceted stress response. Previous studies investigating the effects of stress on $\mathrm{CR}$ have focused on one component of the stress response: cortisol. It was specifically cortisol, examined either by isolating individuals who generated a robust cortisol response to a stress induction (Tsumura et al., 2015) or by directly eliciting a cortisol response using a physiological manipulation (Raio et al., 2013), that was associated with regulatory deficits. Although we observed differences in cortisol responding between the stress and control groups, there was substantial variability that may have reduced our ability to isolate the impact of cortisol specifically on CR. Although male and female participants reported experiencing significant and comparable levels of subjective stress, male participants exhibited a substantially greater cortisol response compared with female participants. The sex specificity of the cortisol response, consistent with previous work (Childs et al., 2010; Kirschbaum et al., 1992, 1999), limited statistical power to examine stress effects in only cortisol responders. Future experiments could focus on the specific effects of cortisol on emotion regulation; such studies would benefit from carefully considering their sample demographics and perhaps testing only male participants, who exhibit a more reliable cortisol response. That said, in this study, cortisol and subjective stress showed substantially different individual patterns-female participants reported marked subjective stress but did not exhibit a significant cortisol response. Thus, focus on cortisol-anchored mechanisms will necessarily steer away from inferences that can generalize to the multifaceted construct of "stress."

It is useful to consider whether stress effects on CR do exist, but they are so subtle that participants could still succeed at the standard CR paradigm despite them. Likert scales lack the sensitivity to capture small quantita- tive changes in affect and are not designed to track qualitative changes in emotional experience. In addition, although the present CR paradigm is the most widely used for neuroimaging, the cognitive processes it examines constitute only one component of what happens when people use CR in daily life. The current study instructed participants to generate one alternative appraisal that effectively reduced their emotional responses. Tsumura and colleagues (2015) instructed participants to generate multiple reappraisals to a negative stimulus under stress and found that cortisol responders generated fewer nonnegative reappraisals. However, the relationship between the number of reappraisals generated and CR effectiveness is currently unknown. It is possible that the moderate level of stress evoked in laboratory studies does indeed reduce the quantity of nonnegative reappraisals an individual can generate, but the participant is still able to generate at least one viable reappraisal and use it to effectively downregulate negative affect. Future work could more thoroughly explore whether the content or range of reappraisals is reduced under acute stress, a possibility that this study was not equipped to evaluate.

CR is believed to be a highly efficacious strategy for regulating emotions, and it is important to understand under what conditions it is effective. Despite mechanistic overlap that suggested otherwise, we found that CR was effective under stress. Although we cannot rule out that there are more subtle or nuanced effects of stress on CR, we demonstrate that a successful multifaceted stress response evoked by a well-validated stress induction did not influence regulation processing during a well-validated CR task. We tentatively conclude that, if stress does influence CR, this effect might be less robust or more circumscribed than expected.

\section{Acknowledgments}

We thank Kate McLaughlin, Kateri McRae, Jens Pruessner, and Jennifer Silvers for helpful discussion and for sharing study materials and Alexandra Rodman, Maggie Schell, Efthalia Kaynor, and Megan Garrad for their help conducting this study. Research reported in this publication was supported through a contract with the U.S. Army Natick Soldier Research, Development, and Engineering Center (Natick, MA) under award number W911QY-14-C-0009 to L. H. S., by the National Science Foundation (DGE1144152 to M. S.), and by the National Institutes of Health Shared Instrumentation Grant (S10OD020039).

Reprint requests should be sent to Leah H. Somerville, Department of Psychology, Harvard University, Northwest Science Building Room 290, 52 Oxford Street, Cambridge, MA 02138, or via e-mail:somerville@fas.harvard.edu.

\section{Note}

1. International Affective Picture System images used: 2038, 2095, 2100, 2102, 2104, 2120, 2205, 2214, 2305, 2357, 2375.1, 2383, 2385, 2393, 2441, 2455, 2480, 2487, 2490, 2493, 2512, 2575, 2579, 2590, 2595, 2661, 2683, 2691, 2700, 2702, 2703, 2710, 2749, 2750, 2799, 2811, 2840, 2870, 2900, 3160, 3180, 
$3220,3280,3300,3350,3500,4605,4621,6211,6212,6250$ 6311, 6312, 6313, 6530, 6555, 6561, 6821, 6840, 7493, 9007, 9070, 9331, 9341, 9404, 9423, 9424, 9429, 9584, 9903, 9905, and 9927.

\section{REFERENCES}

Alexander, J. K., Hillier, A., Smith, R. M., Tivarus, M. E., \& Beversdorf, D. Q. (2007). Beta-adrenergic modulation of cognitive flexibility during stress. Journal of Cognitive Neuroscience, 19, 468-478.

Anisman, H., \& Merali, Z. (1999). Understanding stress: Characteristics and caveats. Alcohol Research \& Health, 23, 241-249.

Arnsten, A. F. T. (2009). Stress signalling pathways that impair prefrontal cortex structure and function. Nature Reviews Neuroscience, 10, 410-422.

Avants, B. B., Tustison, N., \& Song, G. (2009). Advanced normalization tools (ANTS). Insight Journal, 2, 1-35.

Boden, M. T., Bonn-Miller, M. O., Kashdan, T. B., Alvarez, J., \& Gross, J. J. (2012). The interactive effects of emotional clarity and cognitive reappraisal in posttraumatic stress disorder. Journal of Anxiety Disorders, 26, 233-238.

Bradley, M. M., Codispoti, M., Cuthbert, B. N., \& Lang, P. J (2001). Emotion and motivation I: Defensive and appetitive reactions in picture processing. Emotion, 1, 276-298.

Buchanan, T. W., \& Lovallo, W. R. (2001). Enhanced memory for emotional material following stress-level cortisol treatment in humans. Psychoneuroendocrinology, 26, 307-317.

Buhle, J. T., Silvers, J. A., Wage, T. D., Lopez, R., Onyemekwu, C., Kober, H., et al. (2014). Cognitive reappraisal of emotion: A meta-analysis of human neuroimaging studies. Cerebral Cortex, 24, 2981-2990.

Campbell, B. A., Wood, G., \& McBride, T. (1997). Origins of orienting and defensive responses: An evolutionary perspective. In Attention and orienting: Sensory and motivational processes (pp. 41-67). Mahwah, NJ: Lawrence Earlbaum Assoc.

Childs, E., Dlugos, A., \& De Wit, H. (2010). Cardiovascular, hormonal, and emotional responses to the TSST in relation to sex and menstrual cycle phase. Psvchopbysiology 47, 550-559.

Cousijn, H., Rijpkema, M., Qin, S., van Marle, H. J. F., Franke, B., Hermans, E. J., et al. (2010). Acute stress modulates genotype effects on amygdala processing in humans. Proceedings of the National Academy of Sciences. U.S.A., 107, 9867-9872.

Cuthbert, B. N., Bradley, M. M., \& Lang, P. J. (1996). Probing picture perception: Activation and emotion. Psychopbysiology, 33, 103-111.

Dedovic, K., Renwick, R., Mahani, N. K., Engert, V., Lupien, S. J., \& Pruessner, J. C. (2005). The Montreal Imaging Stress Task: Using functional imaging to investigate the effects of perceiving and processing psychosocial stress in the human brain. Journal of Psychiatry and Neuroscience, 30, 319-325.

Denson, T. F., Creswell, J. D., Terides, M. D., \& Blundell, K. (2014). Cognitive reappraisal increases neuroendocrine reactivity to acute social stress and physical pain. Psychoneuroendocrinology, 49, 69-78.

Denson, T. F., Mehta, P. H., \& Ho Tan, D. (2013). Endogenous testosterone and cortisol jointly influence reactive aggression in women. Psychoneuroendocrinology, 38, 416-424.

Goldin, P. R., McRae, K., Ramel, W., \& Gross, J. J. (2008). The neural bases of emotion regulation: Reappraisal and suppression of negative emotion. Biological Psychiatry, 63, 577-586.

Gorgolewski, K., Burns, C. D., Madison, C., Clark, D., Halchenko, Y. O., Waskom, M. L., et al. (2011). Nipype: A flexible, lightweight and extensible neuroimaging data processing framework in Python. Frontiers in

Neuroinformatics, 5, 13.

Greve, D. N., \& Fischl, B. (2009). Accurate and robust brain image alignment using boundary-based registration. Neuroimage, 48, 63-72.

Gross, J. J. (1998). Antecedent- and response-focused emotion regulation: Divergent consequences for experience, expression, and physiology. Journal of Personality and Social Psychology, 74, 224-237.

Gross, J. J., \& John, O. P. (2003). Individual differences in two emotion regulation processes: Implications for affect, relationships, and well-being. Journal of Personality and Social Psychology, 85, 348-362.

Hellhammer, D. H., Wüst, S., \& Kudielka, B. M. (2009). Salivary cortisol as a biomarker in stress research. Psvchoneuroendocrinology, 34, 163-171.

Kirschbaum, C., Kudielka, B. M., Gaab, J., Schommer, N. C., \& Hellhammer, D. H. (1999). Impact of gender, menstrual cycle phase, and oral contraceptives on the activity of the hypothalamus-pituitary-adrenal axis. Psvchosomatic Medicine, 61, 154-162.

Kirschbaum, C., Pirke, K. M., \& Hellhammer, D. H. (1993). The "Trier Social Stress Test"-A tool for investigating psychobiological stress responses in a laboratory setting. Neuropsychobiology, 28, 76-81.

Kirschbaum, C., Wust, S., \& Hellhammer, D. (1992). Consistent sex differences in cortisol responses to psychological stress. Psvchosomatic Medicine, 54, 648-657.

Lang, P. J., Bradley, M. M., \& Cuthbert, B. N. (1997). International Affective Picture System (IAPS): Technical manual and affective ratings (pp. 39-58). Gainesville, FL: NIMH Center for the Study of Emotion and Attention.

Lang, P. J., Greenwald, M. K., Bradley, M. M., \& Hamm, A. O. (1993). Looking at pictures: Affective, facial, visceral, and behavioral reactions. Psychophysiology, 30, 261-273.

Luethi, M., Meier, B., \& Sandi, C. (2009). Stress effects on working memory, explicit memory, and implicit memory for neutral and emotional stimuli in healthy men. Frontiers in Behavioral Neuroscience, 2, 5.

Mai, J. K., Paxinos, G., \& Voss, T. (2008). Atlas of the buman brain (3rd ed.). New York: Academic Press.

McEwen, B. S. (2000). The neurobiology of stress: From serendipity to clinical relevance. Brain Research, 886, 172-189.

Miklósi, M., Martos, T., Szabó, M., Kocsis-Bogár, K., \& Forintos, D. P. (2014). Cognitive emotion regulation and stress: A multiple mediation approach. Translational Neuroscience, 5, 64-71

Moore, S. A., Zoellner, L. A., \& Mollenholt, N. (2008). Are expressive suppression and cognitive reappraisal associated with stress-related symptoms? Behaviour Research and Therapy, 46, 993-1000.

Ochsner, K. N., \& Gross, J. J. (2005). The cognitive control of emotion. Trends in Cognitive Sciences, 9, 242-249.

Ochsner, K. N., Ray, R. D., Cooper, J. C., Robertson, E. R., Chopra, S., Gabrieli, J. D. E., et al. (2004). For better or for worse: Neural systems supporting the cognitive down- and up-regulation of negative emotion. Neuroimage, 23, 483-499.

Olver, J. S., Pinney, M., Maruff, P., \& Norman, T. R. (2015). Impairments of spatial working memory and attention following acute psychosocial stress. Stress and Health, 31, $115-123$.

Payne, J. D., Jackson, E. D., Hoscheidt, S., Ryan, L., Jacobs, W. J., \& Nadel, L. (2007). Stress administered prior to encoding impairs neutral but enhances emotional long-term episodic memories. Learning \& Memorv, 14, 861-868.

Pruessner, J. C., Kirschbaum, C., Meinlschmid, G., \& Hellhammer, D. H. (2003). Two formulas for computation of 
the area under the curve represent measures of total hormone concentration versus time-dependent change. Psvchoneuroendocrinologv, 28, 916-931.

$\mathrm{R}$ Core Team. (2015). R: A language and environment for statistical computing. Vienna: R Foundation for Statistical Computing.

Raio, C. M., Orederu, T. A., Palazzolo, L., Shurick, A. A., \& Phelps, E. A. (2013). Cognitive emotion regulation fails the stress test. Proceedings of the National Academv of Sciences. U.S.A., 110, 15139-15144.

Selye, H. (1987). Stress without distress. London: Transworld.

Sheppes, G., Scheibe, S., Suri, G., \& Gross, J. J. (2011). Emotionregulation choice. Psychological Science, 22, 1391-1396.
Smith, S. M., Jenkinson, M., Woolrich, M. W., Beckmann, C. F., Behrens, T. E. J., Johansen-Berg, H., et al. (2004). Advances in functional and structural MR image analysis and implementation as FSL technical report TR04SS2

Neuroimage, 23, S208-S219.

Tsumura, H., Sensaki, J., \& Shimada, H. (2015). Stress-induced cortisol is associated with generation of non-negative interpretations during cognitive reappraisal. BioPsychoSocial Medicine, 9, 23.

van Marle, H. J. F., Hermans, E. J., Qin, S., \& Fernández, G. (2009). From specificity to sensitivity: How acute stress affects amygdala processing of biologically salient stimuli. Biological Psvchiatry, 66, 649-655. 
This article has been cited by:

1. S. P. Ahmed, L. H. Somerville, C. L. Sebastian. 2017. Using temporal distancing to regulate emotion in adolescence: modulation by reactive aggression. Cognition and Emotion 40, 1-15. [Crossref] 